\title{
Photonic crystal slow light waveguides in a kagome lattice
}

\author{
Sebastian A. Schulz ${ }^{1,2,3,}{ }^{*}$, Jeremy Upham ${ }^{3}$, Liam O'Faolain ${ }^{1,2,4}$, and Robert W. Boyd ${ }^{3,5}$ \\ ${ }^{1}$ Centre for Advanced Photonics and Process Analysis, Cork Institute of Technology, Cork, \\ Ireland. \\ ${ }^{2}$ Tyndall National Institute, Cork, Ireland \\ ${ }^{3}$ Department of Physics, University of Ottawa, 25 Templeton Street, Ottawa, K1N 6N5, \\ Ontario, Canada. \\ ${ }^{4}$ School of Physics and Astronomy, SUPA, University of St Andrews, North Haugh, KY16 \\ 9SS, St Andrews, Scotland, UK. \\ ${ }^{5}$ Institute of Optics and Department of Physics and Astronomy, University of Rochester, \\ Rochester, NY 14627, USA \\ ${ }^{*}$ Corresponding author: sebastian.schulz@cit.ie
}

December 20, 2017

\section{Abstract}

Slow light photonic crystal waveguides tightly compress propagating light and increase interaction times, showing immense potential for all-optical delay and enhanced light-matter interactions. Yet, their practical application has largely been limited to moderate group index values $(<100)$, due to a lack of waveguides that reliably demonstrate slower light. This limitation persists because nearly all such research has focused on a single photonic crystal lattice type: the triangular lattice. Here, we present waveguides based on the kagome lattice that demonstrate an intrinsically high group index and exhibit slow and stopped light. We experimentally demonstrate group index values of $>150$, limited by our measurement resolution. The kagome-lattice waveguides are an excellent starting point for further slow light engineering in photonic crystal waveguides.

Following the discovery of the photonic crystal $(\mathrm{PhC})[1,2]$ the triangular lattice of air holes in a high-refractive-index, semiconductor slab emerged as the most practical realization of $\mathrm{PhCs}$ [3]. The introduction of waveguides of narrow width - generally a single row of holes is removed (W1) - allowed for the demonstrations of PhC-based devices and slow light in $\mathrm{PhC}$ waveguides $[4,5,6,7]$. These early devices were limited by two factors: (1) strong group velocity dispersion in the slow light region and (2) high propagation loss in the slow light region, both due to operation near the band edge. These limitations were overcome with the advent of dispersion engineered $[8,9,10,11]$ and loss engineered waveguides[12, 13], respectively. The resultant technological advances led to a wide range of work on $\mathrm{PhC}$ devices, including tunable delay lines [14], adiabatic control of slow light [15], ultrasmall optical switches [16], enhanced nonlinear effects $[17,18,19,20]$ and enhanced sensitivity spectrometers [21].

Despite this tremendous progress a new limitation has emerged. Photonic crystal waveguides have not reliably demonstrated very high group indices $\left(n_{g}\right)$ (exceeding $\left.n_{g}=100\right)$ [11, 22, 23], with most work 

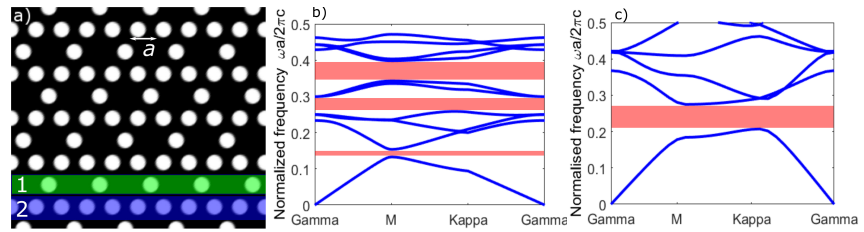

Figure 1: (Colour online) a) Sketch of a kagome lattice of low-index holes in a high-index background. 1 indicates a sparse row, where every second lattice site is missing and 2 indicates a complete row, identical to that of a triangular lattice with the same hole spacing, a. b) Simulated bandstructure of the kagome lattice for TE polarized light. c) Bandstructure of a triangular lattice (TE polarized light) for comparison. In both $\mathrm{b}$ and $\mathrm{c}$ the shaded regions indicate the photonic bandgaps.

being performed either at relatively low $n_{g}$ values $\left(20<n_{g}<50\right)$ or in $\mathrm{PhC}$ cavities, i.e. a standing wave system. A new approach is needed to achieve higher group indices, spanning the wide gap between current slow light waveguides and cavities, to realize the full potential of $\mathrm{PhC}$ based slow light devices. The solution that we present here is $\mathrm{PhC}$ waveguides based on the kagome lattice. These waveguides have intrinsically high group indices and serve as a fresh starting point for further slow light engineering. We will first describe the kagome lattice and outline why it is particularly well suited for slow light waveguides. This will be followed by a description of line defect waveguides in the kagome lattice and an experimental demonstration of slow light $\left(n_{g}>150\right)$ obtained with only limited waveguide engineering.

Any new $\mathrm{PhC}$ lattice has to satisfy the following conditions. It has to allow for realistic devices, i.e. a connected membrane or substrate supported structure with minimum dimensions that are attainable with current fabrication techniques; it has to support an optical bandgap, to allow the introduction of guided modes; and, most importantly, it should outperform the traditional triangular lattice with respect to the figure of merit under consideration, here the group index.

The kagome lattice fulfills all these conditions. It is well known in the solid state physics [24] and $\mathrm{PhC}$

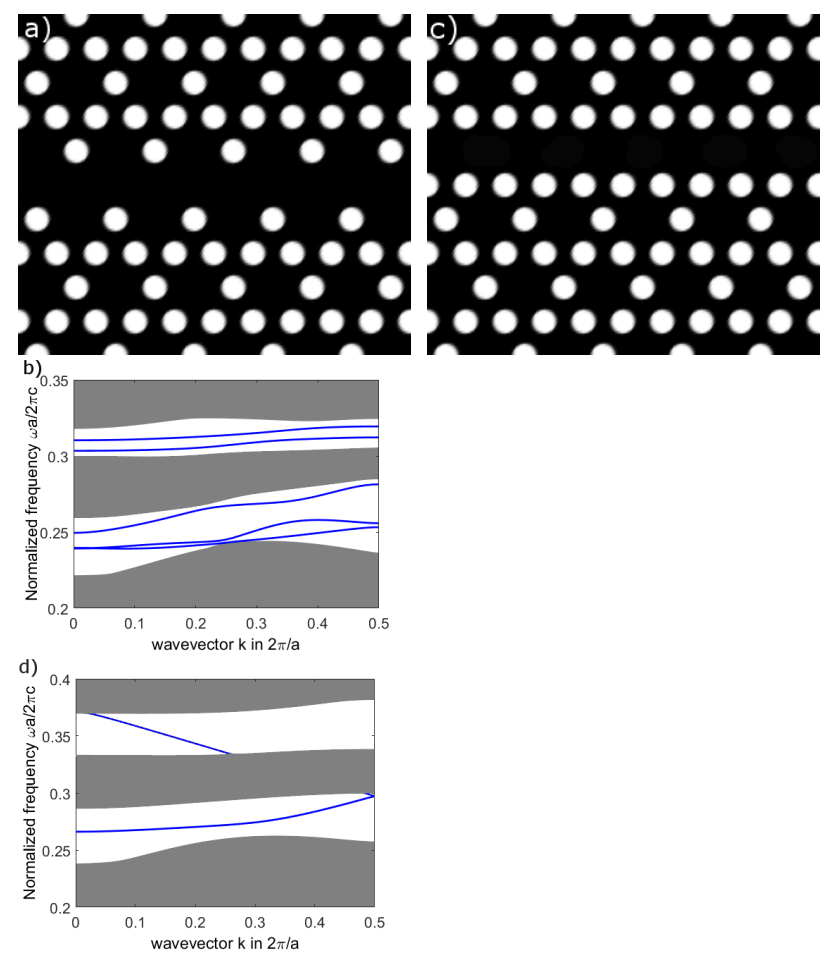

Figure 2: (Colour online) Sketch of different waveguides in a kagome lattice and their respective bandstructure. a) is formed by removing a complete row, with the bandstructure shown in b). c) is formed by removing a sparse row and the corresponding bandstructure is shown in d). In the bandstructure plots blue indicates a waveguide mode, white indicates the photonic band gap regions and grey shading indicates bulk modes. 
fibre [25] communities, but has attracted only limited interest for PhC slabs [26, 27, 28]. The kagome lattice is a depleted version of the triangular lattice, consisting of a sparse super-lattice imposed on the standard triangular lattice. As shown in Fig. 1a, along every second diagonal line of a triangular lattice each second lattice site is removed to form the kagome lattice.

Intuitively, the kagome lattice is better represented by a tight-binding model, compared to the triangular lattice which is better approximated by a "free photon" (or free electron for electronic crystals) approximation. This leads to larger photonic band gaps and flatter bands in the kagome lattice [27, 29]. If we consider a lattice of air holes in a $220 \mathrm{~nm}$-thick silicon slab, the material system used for most slow light $\mathrm{PhC}$ work, the kagome lattice supports multiple optical bandgaps for TE polarized light, as shown in Fig. 1b. Furthermore, the sparse super-lattice results in a "compressed' bandstructure, with each mode occupying a narrower frequency range compared to the triangular lattice. For example, the first bandgap of the kagome lattice starts at a normalized frequency of 0.15 , while that of a triangular lattice with the same lattice parameters starts at a normalized frequency of 0.21 , see Fig. 1. The compressed bandstructure thus exhibits a reduced slope for all optical modes, resulting in an increased group index, since the group index is defined as:

$$
n_{g}=\frac{d k}{d \omega} .
$$

Therefore, the kagome lattice naturally lends itself to applications requiring flat optical bands, including slow light waveguides.

Kagome-lattice waveguides are formed in the same way as traditional $\mathrm{PhC}$ waveguides, through the removal of a single row of holes. The increased complexity of the kagome lattice however allows for a larger variety of waveguides. We can define two types of rows of holes in the kagome lattice: complete rows, where all holes are present, and sparse rows, which include the lattice sites that have been removed to form the kagome lattice, as shown in Fig. 1a . The removal of either a complete or a sparse row, along any crystal axis, will result in a waveguide, as shown in Fig. 2. Preliminary simulations, performed using the MIT photonics band package [30] (Fig. 1b and d) indicate that all of these waveguides can support guided modes, providing a vastly increased design space compared to the classical triangular lattice.

Many of these waveguides merit further study, but, for the remainder of this article, we will focus on a single configuration (Fig. 3). We chose this waveguide for its intriguing potential for slow light, as demonstrated by the shallow slope of the optical modes shown in Fig. 3a.

The waveguide that we are considering is formed by removing a complete row, i.e. one with no missing holes, and subsequently shifting the two $\mathrm{PhC}$ claddings by $a / 2$ with respect to each other. The bandstructure of this waveguide supports optical modes in two bandgaps in the normalized frequency range considered here, $0.25 \leq \omega \leq 0.36$, see Fig. 3.a. Closer inspection shows that the modes in the upper bandgap have the desired shallow slope, indicating high group indices. Since our goal is to reach group indices unattainable in the triangular lattice, these modes are of particular interest to us and we now focus on the upper bandgap. Here, two optical modes have a shallow slope, i.e. a large group index, below the light line.

The field distributions within these modes (see Figs. 3b and c) are unusual, not matching those of a triangular-lattice $\mathrm{PhC}$ waveguide $[12,31]$. Instead, they suggest an intermediate behaviour, between a traditional waveguide and a coupled cavity waveguide. Within each unit cell, the field distribution closely resembles that of a $\mathrm{PhC}$ cavity, yet the system is open along the $\mathrm{x}$-axis and the field can propagate through the waveguide. Once again, an intuitive explanation of this is that the field is tightly bound within one unit cell, but can still propagate along the device, i.e. a tight binding model is best suited to describe this system.

To verify the slow light behaviour, we fabricated kagome lattice $\mathrm{PhC}$ waveguides in an air-bridged silicon membrane geometry, Fig. 4a. The pattern was defined in a ZEP-520A resist layer, through electron beam lithography (Raith Pioneer, 30kV) and transferred into the silicon slab by a reactive ion dry etch $\left(\mathrm{SF}_{6} / \mathrm{CHF}_{3}\right.$ gas mixture). After stripping of the resist, windows were opened in S1811 photoresist by 

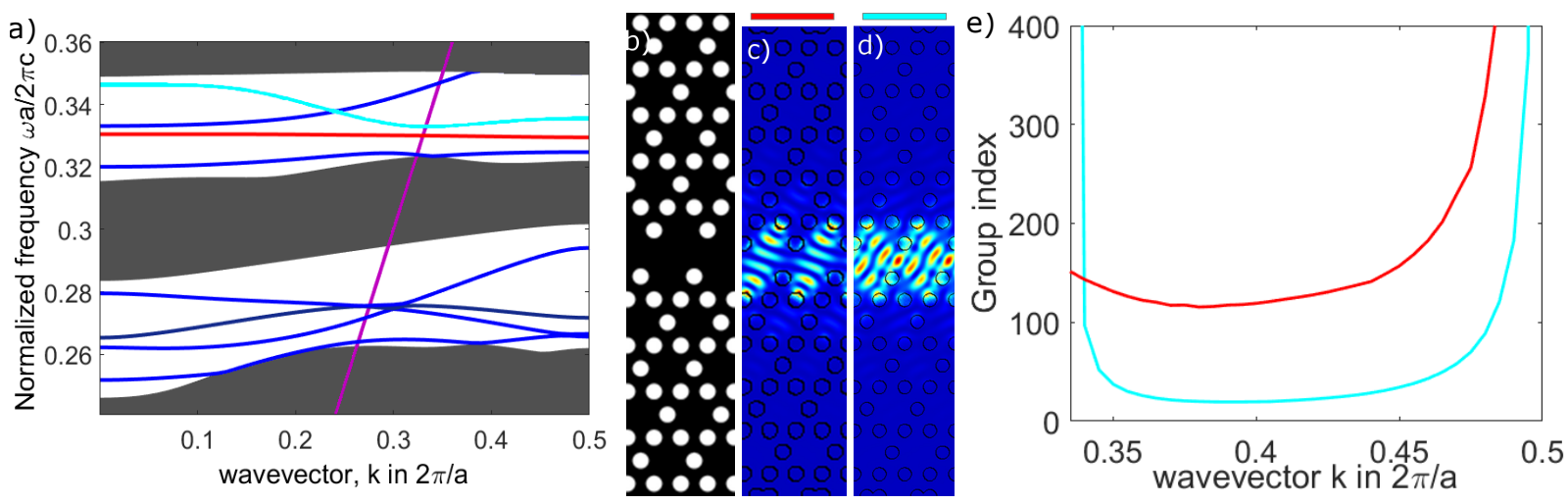

Figure 3: (Colour online) a) Dispersion curve of the waveguide under investigation. The modes of interest, lying in the second bandgap, are highlighted in red and light blue. The light line is shown in purple. b) Sketch of a section of the kagome-lattice waveguide. The waveguide width is conventionally given as wa $\sqrt{3}$, as indicated in the sketch. In our case $w=0.8$ and $a=500 \mathrm{~nm}$. c) and d) representative field distributions of the optical modes shown in a) as red and light blue, respectively, plotted for $k=0.4$. The field profiles differ significantly from that of a traditional triangular-lattice waveguide and indicate an intermediate behaviour, between a traditional waveguide and a cavity based system, providing an intuitive understanding of the origin of the slow light in this waveguide. e) Group index of the two waveguide modes plotted against wavevector. Only the guided mode region, below the lightline, is shown. The two modes have a minimum group index of 20 and 125 respectively.

optical lithography. The buried oxide was then removed through a hydrofluoric acid wet etch, creating an air-bridge $\mathrm{PhC}$ membrane. The as fabricated $\mathrm{PhC}$ parameters are: a lattice period of $520 \mathrm{~nm}$, a hole radius of $138 \mathrm{~nm}$, a central waveguide width of $w=0.8$ and a slab thickness of $h=201 \mathrm{~nm}$. The radius and lattice constant are larger than that of a triangular-lattice waveguide, indicating that traditional fabrication methods are well suited for kagomelattice waveguides. Optical characterization was performed using a broadband (1525-1575 nm) amplified spontaneous emission source, coupled to the sample through grating couplers operating at 10-degree incidence [32]. The transmitted light was analyzed using a fibre-coupled optical spectrum analyser, with a resolution of $0.02 \mathrm{~nm}$, and normalized to a reference waveguide without a PhC. For the group index measurements the sample is placed in one arm of an unbalanced Mach-Zehnder interferometer and the resulting interference spectrum is analysed according to Ref. [33]. The setups for both the transmission and group index measurements are shown in more detail in Ref. [34]. The resulting transmission and group index spectra are shown in Fig. $4 \mathrm{~b}$ and c.

The experimental data (Fig. $4 \mathrm{~b}$ and c) shows two high group index peaks, with $n_{g}>150$. The central wavelength of the peaks is separated by about $15 \mathrm{~nm}$ and the intermediate region shows a modest group index $\left(n_{g} \approx 20\right)$. This experimental group index spectrum matches well with the theoretically predicted group index curve for this waveguide (dashed line in Fig. 4c). In fact, from a closer examination of the calculated bandstructure, Fig. 4d, we can see that both group index peaks are associated with stationary points in the bandstructure, i.e. a zero slope, indicating that the observed values are limited by the resolution of the measurement apparatus, with the real group index corresponding to stopped light.

Our simulations, supported by experimental observations, show that the kagome lattice supports optical modes with extremely slow and stopped light away from the bandedge - even before the applica- 

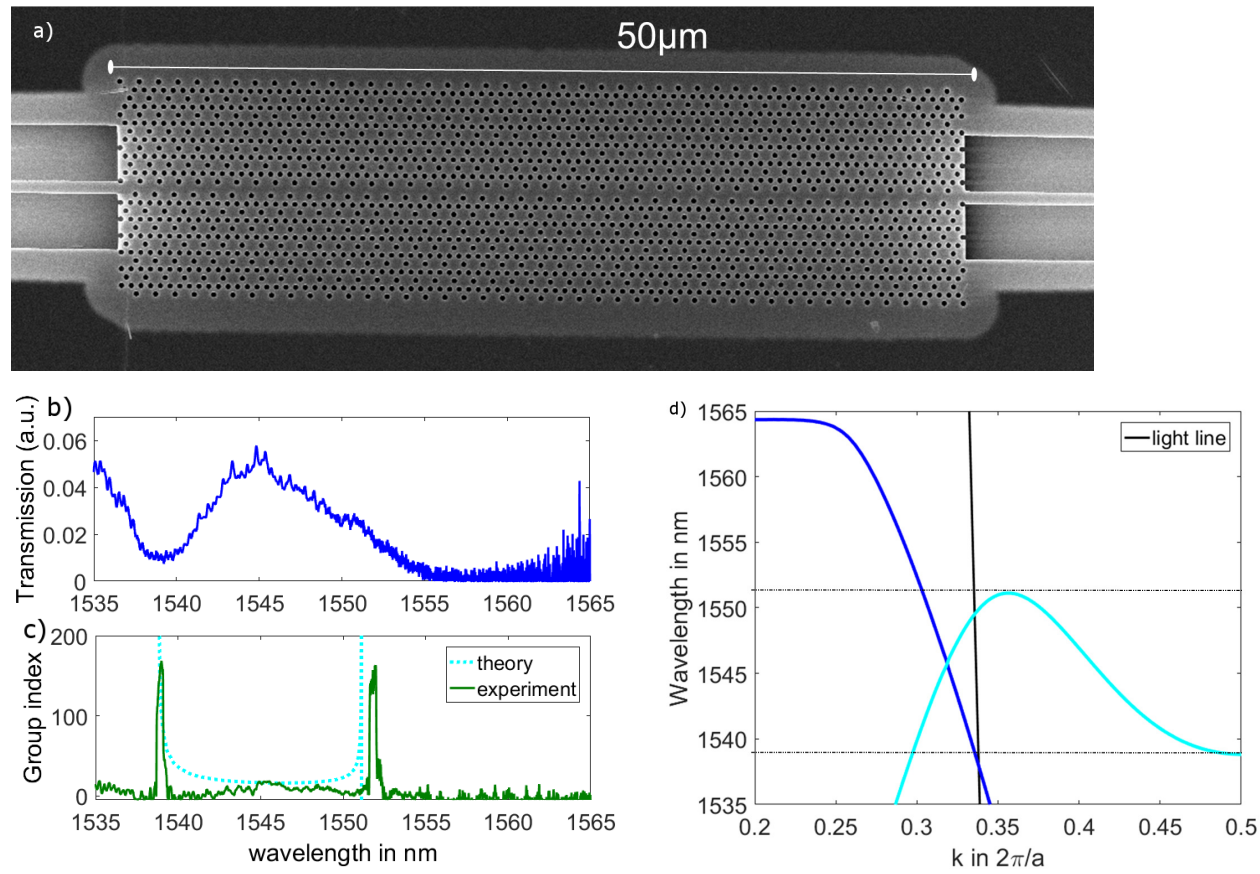

Figure 4: (Colour online) a) Scanning electron microscope image of an air-bridge kagome-lattice PhC waveguide. b) Experimental transmission spectrum of the waveguide shown in a). c) Experimental (solid, green) and theoretical (dashed, light blue) group index spectra. The corresponding bandstructure is shown in d). The solid black line indicates the light line and the dark blue line an additional mode that co-exist in the observed wavelength region. The stationary points in the bandstructure occur at the same wavelength as the peaks in the group index spectra (indicated by the black dashed lines). Therefore, both the experimental and theoretical group index are limited by the measurement/simulation resolution and the high group index peaks are associated with stopped light, which is occurring below the light line and - for the high wavelength peak - away from the bandedge $(k=0.36)$. 
tion of dispersion engineering. As shown earlier in Fig. 3, two modes are supported within the second bandgap, with our experiment limited to observe only the upper (higher frequency) mode, with the second mode being located at a wavelength outside of our source range, due to the difference between the design and actual slab thickness $(220 \mathrm{~nm}$ and $201 \mathrm{~nm}$, respectively).

To summarise, we have introduced the concept and designs of $\mathrm{PhC}$ waveguides in a kagome lattice. Out of this vast new design space we further investigated a single waveguide design. We experimentally observe group indices exceeding 150, limited by our measurement resolution and associated with stationary points in the bandstructure. We believe that kagome-lattice waveguides present a huge new parameter space for $\mathrm{PhC}$ design, enabling extreme group index values and the study of new phenomena in waveguides, bridging the gap between traditional $\mathrm{PhC}$ waveguides, $\mathrm{PhC}$ cavities and coupled cavity waveguides.

\section{Funding}

This research was funded by the Canada Excellence Research Chair (CERC) program and L. O'Faolain and S. A. Schulz acknowledge support from the European Research Council Starting grant no. 337508.

\section{Acknowledgments}

We thank K. Tsakmakidis for fruitful discussions.

\section{References}

[1] E. Yablonovitch, "Inhibited spontaneous emission in solid-state physics and eelectronics," Physical Review Letters 58, 2059-2062 (1987).

[2] S. John, "Strong localization of photons in certain disordered dielectric superlattices," Physical Review Letters 58, 2486-2489 (1987).

[3] T. F. Krauss, R M De La Rue, and S. Brand, "Two-dimensional photonic-bandgap structures operating at near-infrared wavelengths," Nature 383, 699 (1996).

[4] M. Notomi, K. Yamada, A. Shinya, J. Takahashi, C. Takahashi, and I. Yokohama, "Extremely large group-velocity dispersion of linedefect waveguides in photonic crystal slabs," Phys. Rev. Lett. 87, 253902 (2001).

[5] T.F.Krauss, "Why do we need slow light?" Nature Photonics 2, 448 (2008).

[6] T. Baba, "Slow light in photonic crystals," Nature Photonics 2, 465 (2008).

[7] T.F.Krauss, "Slow light in photonic crystal waveguides," J. Phys. D: Appl. Phys. 40, 2666 (2007).

[8] L.H.Frandsen, A.V.Lavrinenko, J.FagePedersen, and P.I.Borel, "Photonic crystal waveguides with semi-slow light and tailored dispersion properties," Optics Express 14, 9444 (2006).

[9] J. Li, T. P. White, L. O'Faolain, A. GomezIglesias, and T. F. Krauss, "Systematic design of flat band slow light in photonic crystal waveguides," Optics Express 16, 6227-6232 (2008).

[10] D. Mori and T. Baba, "Wideband and low dispersion slow light by chirped photonic crystal coupled waveguide," Optics Express 13, 9398 (2005).

[11] S. A. Schulz, L. O'Faolain, D. M. Beggs, T. P. White, A. Melloni, and T. F. Krauss, "Dispersion engineered slow light in photonic crystals: a comparison," Journal of Optics 12, 104004 (2010).

[12] L. O'Faolain, S. A. Schulz, D. M. Beggs, T. P. White, M. Sapsenovic, L. Kuipers, F. Morichetti, A. Melloni, S. Mazoyer, J. P. Hugonin, P. Lalanne, and T. F. Krauss, "Loss engineered slow light waveguides," Opt. Express 18, 27627 (2010). 
[13] J. Li, L. O'Faolain, S. A. Schulz, and T. F. Krauss, "Low loss propagation in slow light photonic crystal waveguides at group indices up to 60," Photonics and Nanostructures - Fundamentals and Applications 10, 589 (2012).

[14] T. Baba, T. Kawasaki, H. Sasaki, J. Adachi, and D. Mori, "Large delay-bandwidth product and tuning of slow light pulse in photonic crystal coupled waveguide," Optics Express 16, 9245 (2008).

[15] D. M. Beggs, T. F. Krauss, L. Kuipers, and T. Kampfrath, "Ultrafast tilting of the dispersion of a photonic crystal and adiabatic spectral compression of light pulses," Physical Review Letters 108, 033902 (2012).

[16] D. M. Beggs, T. P. White, L. O'Faolain, and T. F. Krauss, "Ultracompact and low-power optical switch based on silicon photonic crystals," Opt. Lett. 33, 147 (2007).

[17] J. Li, L. O'Faolain, I. H. Rey, and T. F. Krauss, "Four-wave mixing in photonic crystal waveguides: slow light enhancement and limitations," Opt. Express 19, 4458-4463 (2011).

[18] B. Corcoran, C. Monat, C. Grillet, D. J. Moss, B. J. Eggleton, T. P. White, L. O'Faolain, and T. F. Krauss, "Green light emission in silicon through slow-light enhanced third-harmonic generation in photonic-crystal waveguides," Nature Photonics 3, 206 (2009).

[19] C. Monat, M. de Sterke, and B. J. Eggleton, "Slow light enhanced nonlinea optics in periodic strucures," Journal of Optics 12, 104003 (2010).

[20] P. Colman, C. Husko, S. Combrie, I. Sagnes, C. W. Wong, and A. De Rossi, "Temporal solitons and pulse compression in photonic crystal waveguides," Nature Photonics 4, 862 (2010).

[21] O. S. Magaa-Loaiza, B. Gao, S. A. Schulz, K. M. Awan, J. Upham, K. Dolgaleva, and R. W. Boyd, "Enhanced spectral sensitivity of a chipscale photonic-crystal slow-light interferometer," Optics Letters 41, 1431-1434 (2016).
[22] Y. Zhao, Y.-N. Zhang, Q. Wang, and H. Hu, "Review on the optimization methods of slow light in photonic crystal waveguide," IEEE Transactions on Nanotechnology 14, 407 (2015).

[23] M. Minkov and V. Savona, "Wide-band slow light in compact photoonic crystal coupledcavity waveguides," Optica 2, 631-634 (2015).

[24] A. Chubukov, "Order from disorder in a kagome antiferromagnet," Physical Review Letters 69, 832 (1992).

[25] P. S. J. Russel, P. Hoelzer, W. Chang, and J. C. Travers, "Hollow-core photonic crystal ffibre for gas-based nonlinear optics," Nature Photonics 8, 278-286 (2014).

[26] H. Takeda, T. Takashia, and K. Yoshino, "Flat photonic bands in two-dimensional photonic crystals with kagome lattices," Journal of Physics: Condensed Matter 16, 6317-6324 (2004).

[27] A. J. Garcia-Adeva, "Band gap atlas for photonic crystals having the symmetry of the kagome and pyroclore lattices," New Journal of Physics 8, 86 (2006).

[28] A. V. Giannopoulos, Y.-J. Li, C. M. Long, J.-M. Jin, and K. D. Choquette, "Optical properties of photonic crystal heterostructure cavity lasers," Optics Express 17, 5379-5390 (2009).

[29] Y. Zhong, S. Xia, L. Tang, D. Song, Y. Hu, Y. Pei, J. Su, Y. Li, and Z. Chen, "Observation of localized flat-band states in kagome photonic lattice." Optics Express 24, 8877-8885 (2016).

[30] S. Johnson and J. Joannopoulos, "Blockiterative frequency-domain methods for maxwell's equations in a planewave basis," Opt. Express 8, 173-190 (2001).

[31] M. Patterson, S. Hughes, S. Schulz, D. M. Beggs, T. P. White, L. O'Faolain, and T. F. Krauss, "Disorder-induced incoherent scattering losses in photonic crystal waveguides: Bloch mode reshaping, multiple scattering, and breakdown of 
the beer-lambert law," Physical Review B 80, 195305 (2009).

[32] D. Taillaert, W. Bogaerts, P. Bienstman, T. F. Krauss, P. V. Daele, I. Moerman, S. Verstuyft, K. D. Mesel, and R. Baets, "An out-of-plane grating coupler for efficient butt-coupling between compact planar waveguides and singlemode fibers," IEE Journal of Quantum Electronics 38, 949 (2002).

[33] A. Gomez-Iglesias, D. O'Brien, L. O'Faolain, A. Miller, and T. F. Krauss, "Direct measurement of the group index of photonic crystal waveguides via fourier transform spectral interferometry," Appl. Phys. Lett. 90, 261107 (2007).

[34] K. M. Awan, S. A. Schulz, D. X. Liu, K. Dolgaleva, J. Upham, and R. W. Boyd, "Post-process wavelength tuning of silicon photonic crystal slow-light waveguides," Optics Letters 40, 19521955 (2015). 